\title{
Supporting Information \\ High-Precision Single-Molecule Identification \\ Based on Single-Molecule Information within a Noisy Matrix
}

Masateru Taniguchi, Takahito Ohshiro, Yuki Komoto, Takayuki Takaai, Takeshi Yoshida, and Takashi Washio

The Institute of Scientific and Industrial Research, Osaka University, 8-1 Mihogaoka, Ibaraki, Osaka 567-0047.

The Supporting Information includes:

1. PUC (Figure S1)

2. Cross-validation (Figure S2)

3. Undersampling (Figure S3)

4. Confusion matrixes for 2-type base molecules (Figure S4)

5. Confusion matrixes for 3-type base molecules (Figure S5)

6. Confusion matrixes for 4-type base molecules (Figure S6)

7. Numbers of extracted pulses (Table S1) 
1. PUC

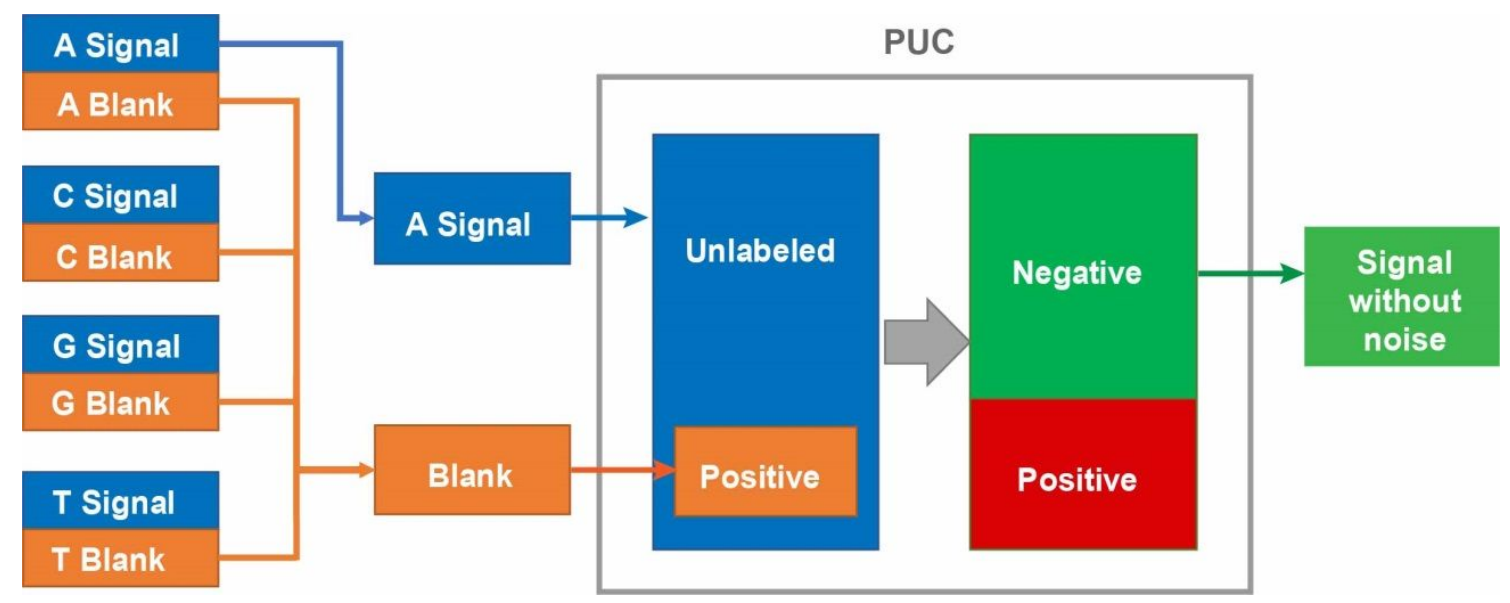

Figure S1. Schematic of PUC. A, C, G and T show deoxyadenosine monophosphate, deoxycytidine monophosphate, deoxyguanosine monophosphate and thymidine monophosphate, respectively. 


\section{Cross-validation}

A

C

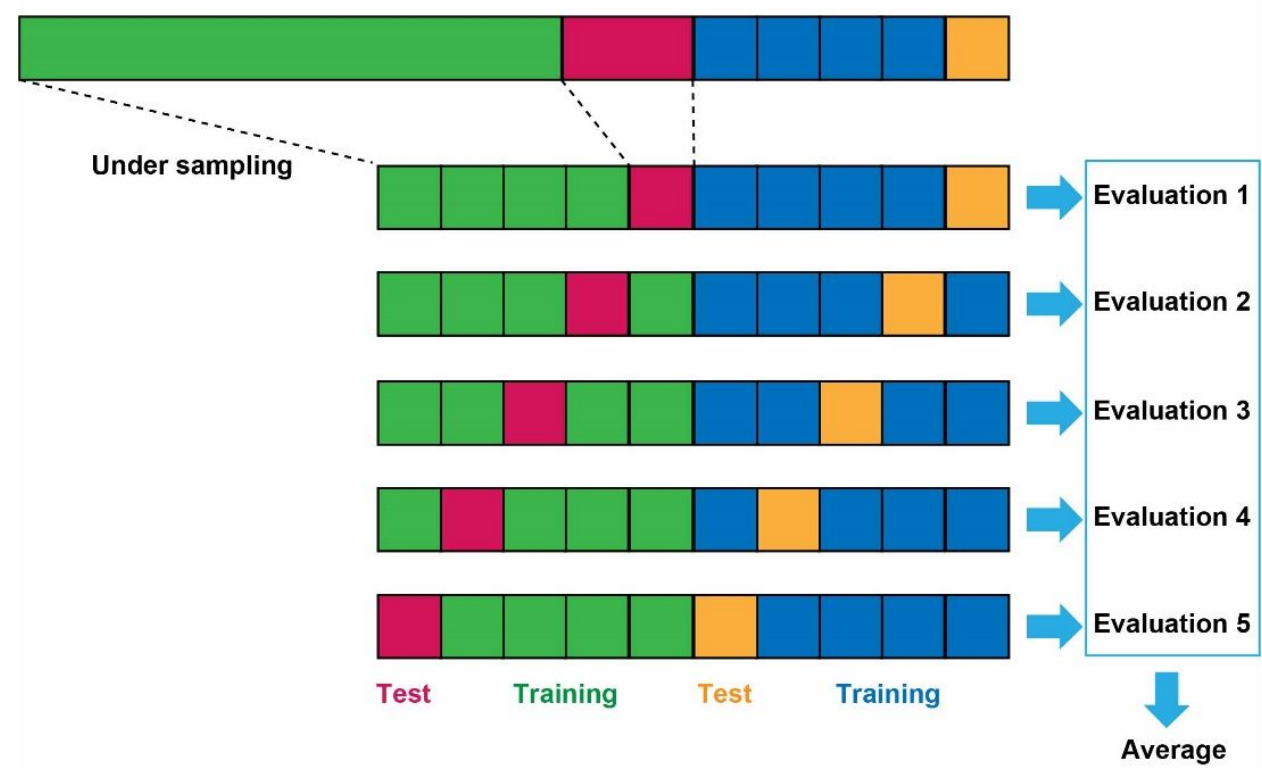

Figure S2. Schematic of $\mathbf{5}$-fold cross-validation. This illustrates the identification of deoxyadenosine monophosphate (A) and deoxycytidine monophosphate $(C)$. 


\section{Undersampling}

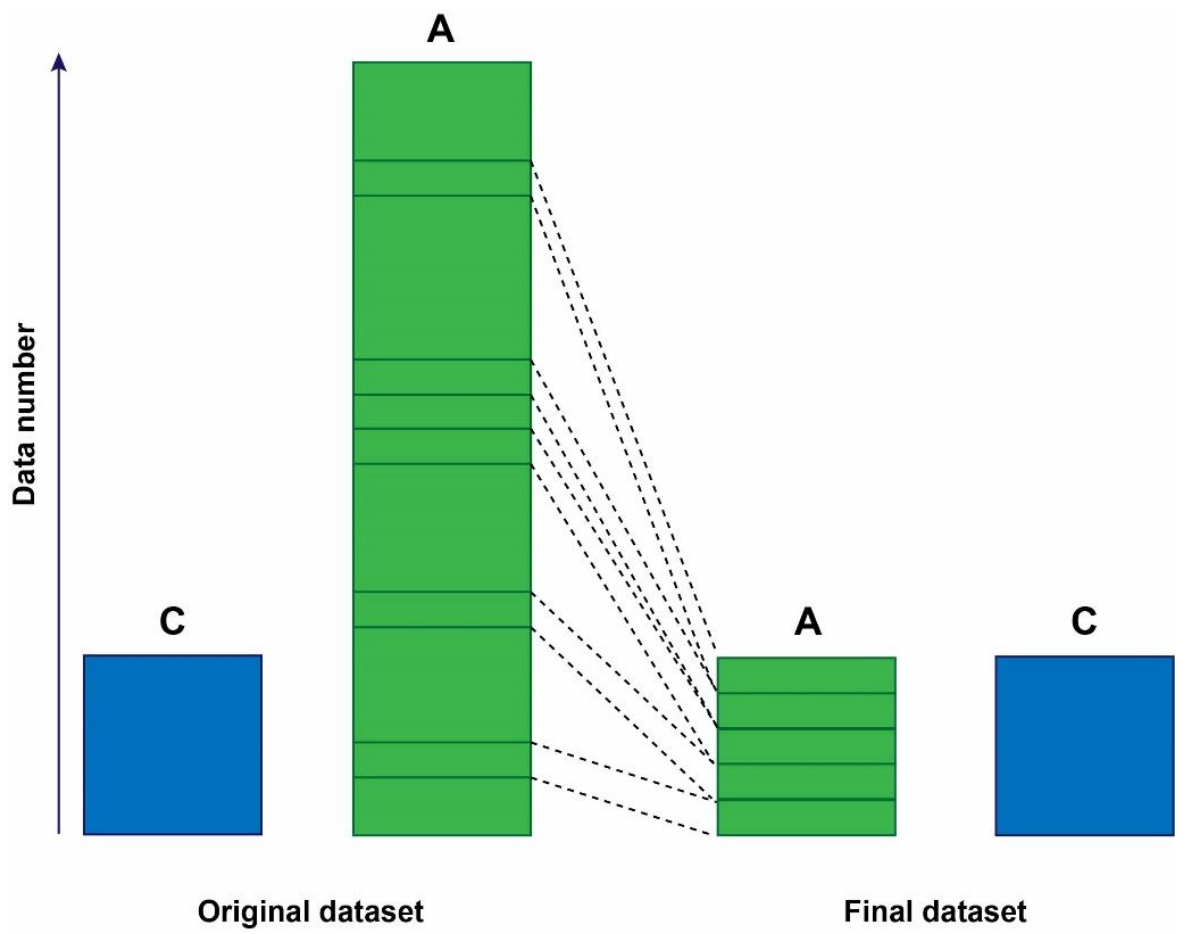

Figure S3. Schematic of undersampling. This illustrates the identification of deoxyadenosine monophosphate (A) and deoxycytidine monophosphate (C). The numbers of the current-time profiles obtained are listed in Table 1. 
(a) Column

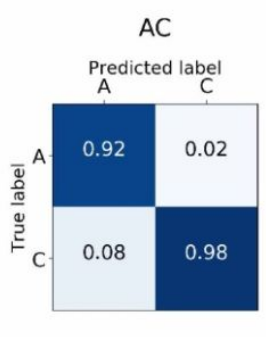

AG

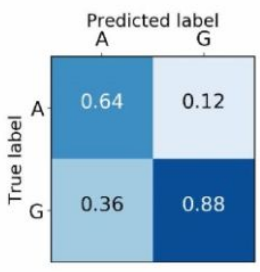

AT

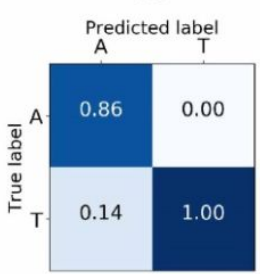

CG

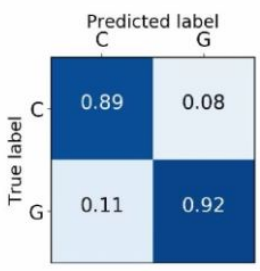

CT

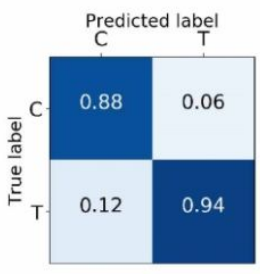

GT

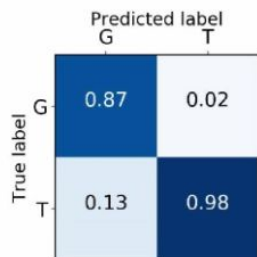

Row

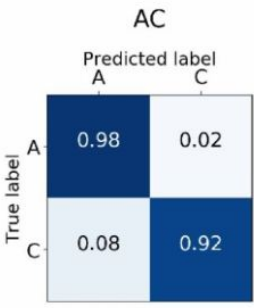

AG

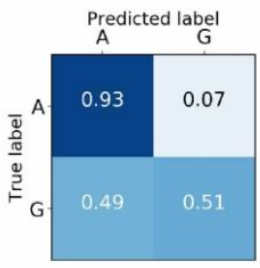

AT

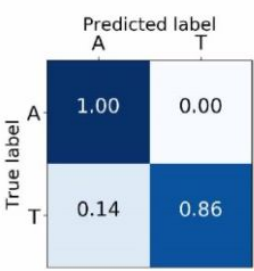

CG

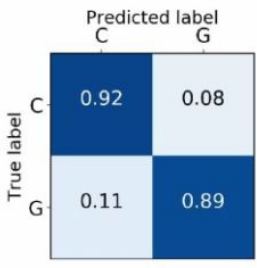

CT

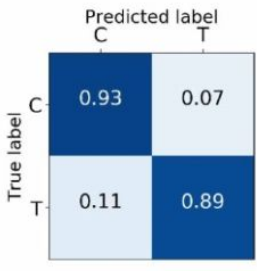

GT

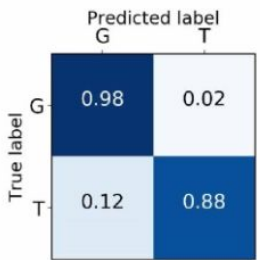

(b) Column

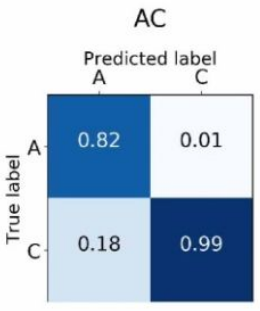

AG

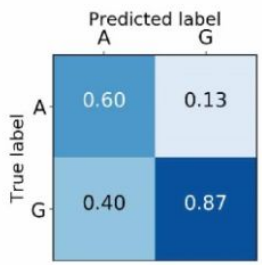

AT

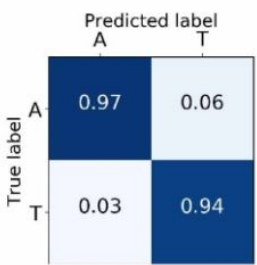

CG

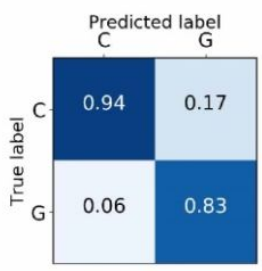

CT

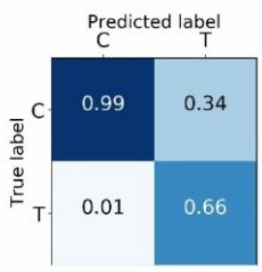

GT

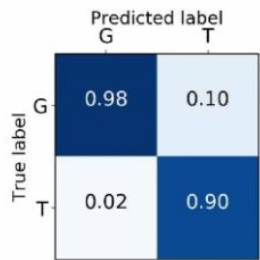

Row

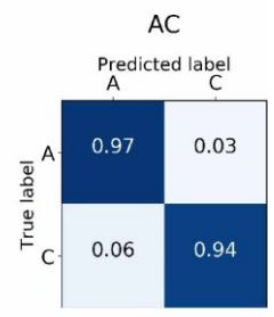

AG

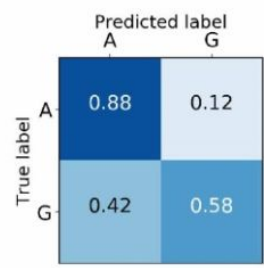

AT

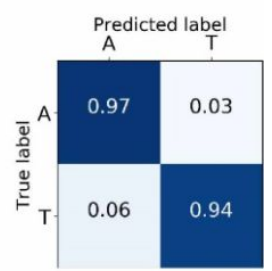

CG

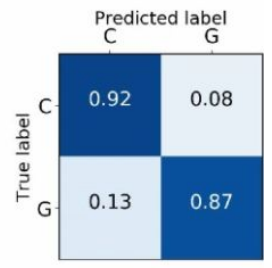

CT

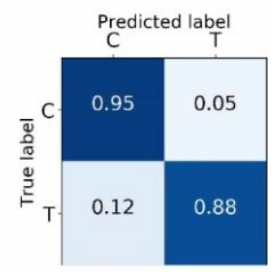

GT

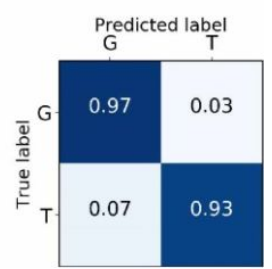

Figure S4. Confusion matrixes for 2-type base molecules (a) without and (b) with PUC. The left and right confusion matrixes are normalised in the columns and rows, respectively. 
(a) Column

ACG

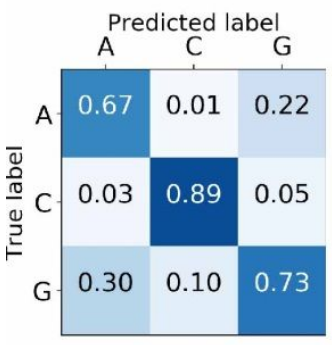

ACT

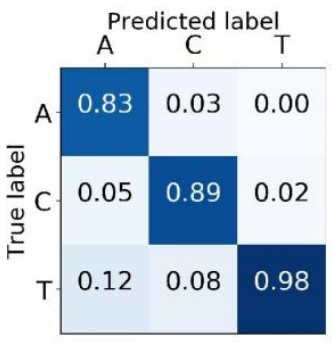

AGT

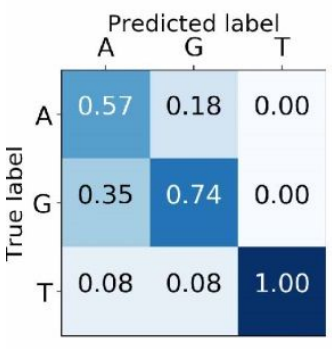

CGT

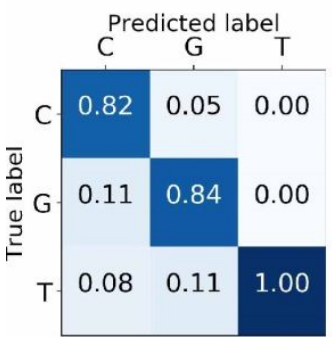

Row

ACG

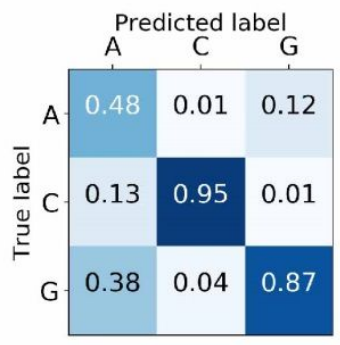

ACT

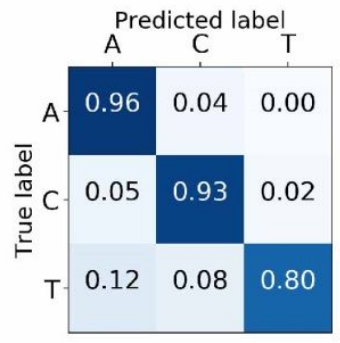

AGT

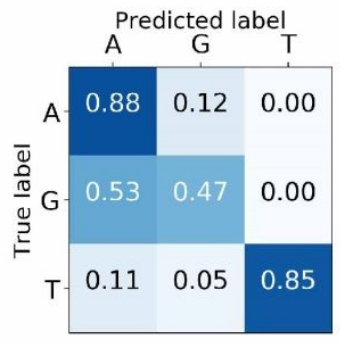

CGT

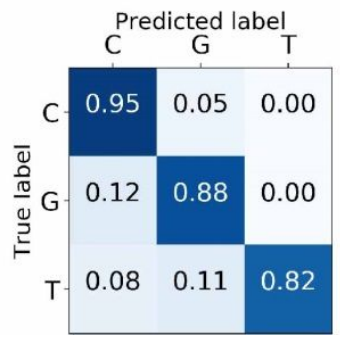

(b)

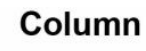

ACG

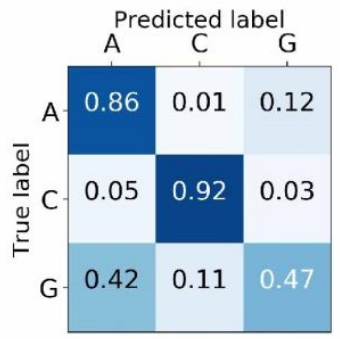

ACT

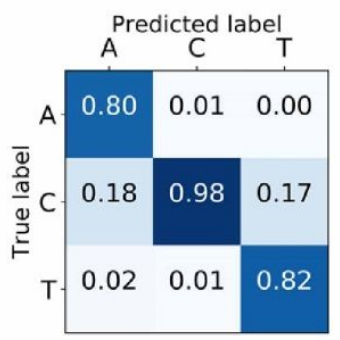

AGT

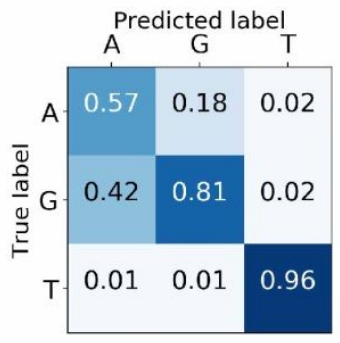

CGT

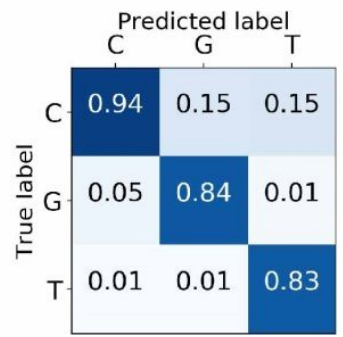

Row

ACG

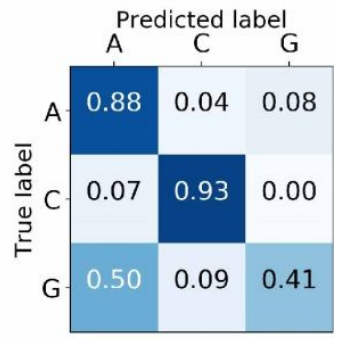

ACT

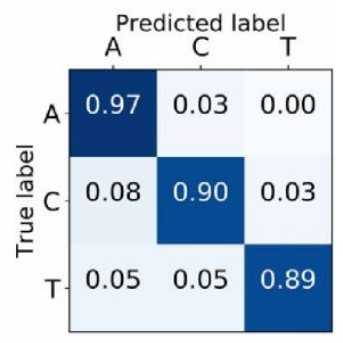

AGT

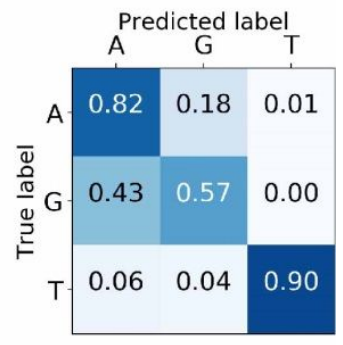

CGT

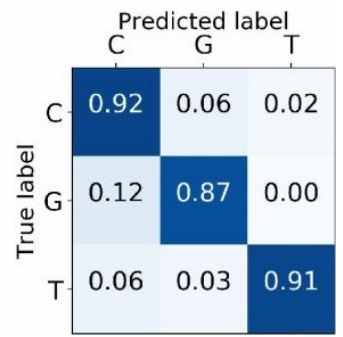

Figure S5. Confusion matrixes for 3-type base molecules (a) without and (b) with PUC. The left and right confusion matrixes are normalised in the columns and rows, respectively. 
(a)

\section{Column}

Row

(b)

Column

Row

$0.54 \mathrm{~nm}$

AGTC

AGTC

A Predicted label

A 0.690 .000 .140 .02

$\begin{array}{lllllll}\bar{\Phi} & 0.01 & 0.90 & 0.08 & 0.00\end{array}$

\begin{tabular}{lllllll}
\hline \multirow{2}{*}{$\mathrm{T}$} & 0.26 & 0.03 & 0.76 & 0.02
\end{tabular}

C $\begin{array}{llll}0.04 & 0.07 & 0.03 & 0.97\end{array}$

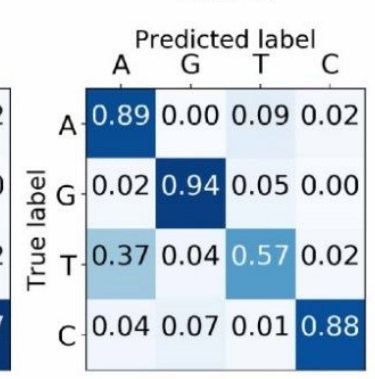

$0.56 \mathrm{~nm}$

ACGT

ACGT

Predicted label

A 0.410 .000 .350 .00

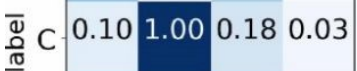

$\begin{array}{llllll}\text { ᄅ르 G } & 0.48 & 0.00 & 0.47 & 0.00\end{array}$

T $0.000 .00 \quad 0.000 .97$

Predicted labe A C G T

A 0.500 .000 .500 .00

$\begin{array}{llllll}\bar{\Phi}_{\pi} C-0.12 & 0.62 & 0.23 & 0.04\end{array}$

$\begin{array}{lllll} & 0.47 & 0.00 & 0.53 & 0.00\end{array}$

T- $0.000 .000 .00 \quad 1.00$

$0.58 \mathrm{~nm}$

ACGT

ACGT
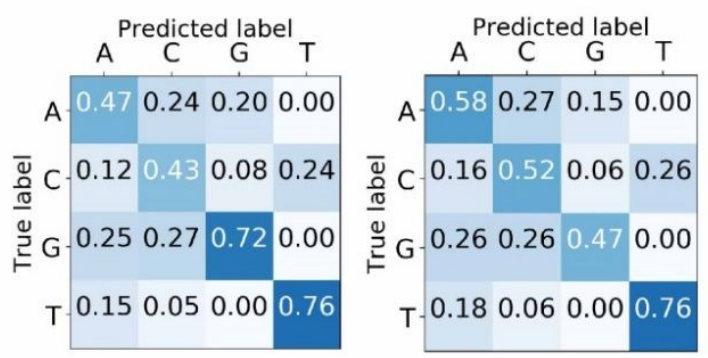

$0.60 \mathrm{~nm}$

ACGT

ACGT

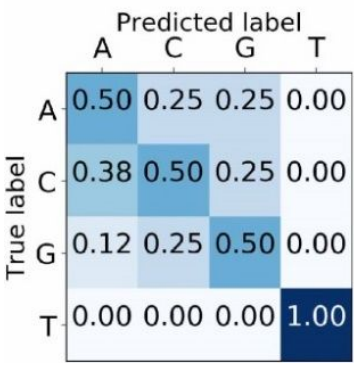

Predicted lab

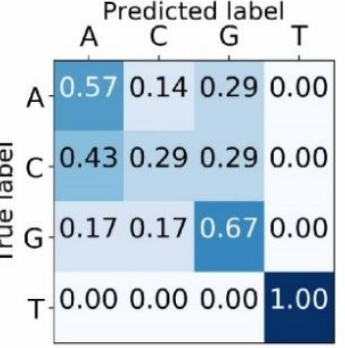

ACGT

$0.54 \mathrm{~nm}$

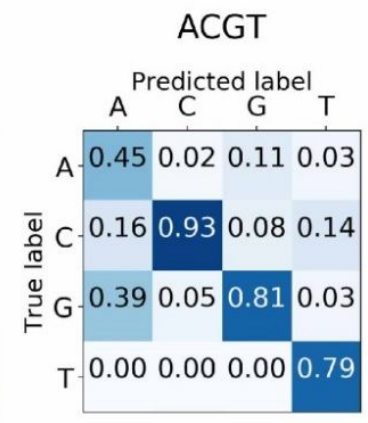

$0.56 \mathrm{~nm}$

\section{ACGT}

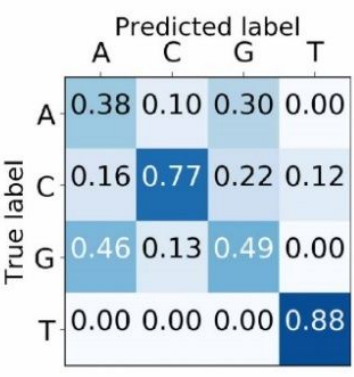

$0.58 \mathrm{~nm}$

ACGT

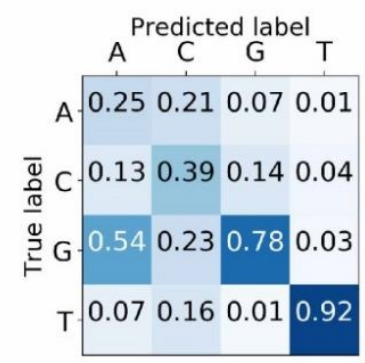

$0.60 \mathrm{~nm}$

\section{ACGT}

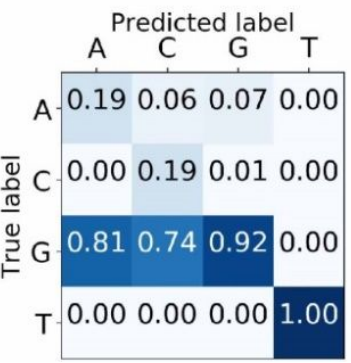

ACGT

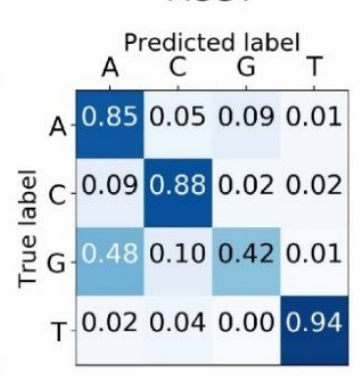

ACGT

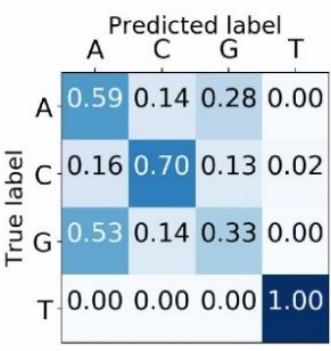

ACGT

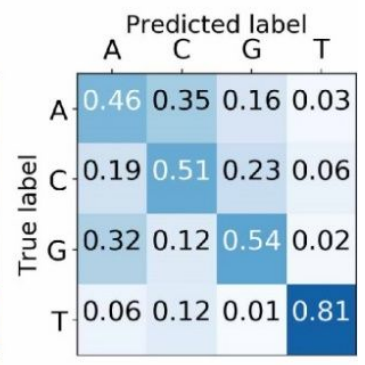

ACGT

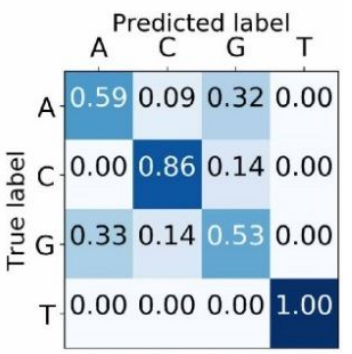

Figure S6. Confusion matrixes for 4-type base molecules(a) without and (b) with PUC. The left and right confusion matrixes are normalised in the columns and rows, respectively. 
Table S1. Numbers of the extracted pulses in blank and single-molecule measurements (which were performed at specific electrode gap distances). The numbers of the extracted pulses show the number of pulses extracted after PUC processing. A, C, G and $\mathrm{T}$ represent deoxyadenosine monophosphate, deoxycytidine monophosphate, deoxyguanosine monophosphate and thymidine monophosphate, respectively.

\begin{tabular}{cccccc}
\hline & Gap $(\mathbf{n m})$ & A & C & G & T \\
\hline \multirow{4}{*}{ Signal } & 0.54 & 787 & 2775 & 1223 & 301 \\
& 0.56 & 522 & 895 & 759 & 135 \\
& 0.58 & 169 & 271 & 584 & 482 \\
& 0.60 & 151 & 70 & 754 & 31 \\
\hline \multirow{4}{*}{ Blank } & 0.54 & 3 & 824 & 28 & 1 \\
& 0.56 & 16 & 176 & 55 & 30 \\
& 0.58 & 0 & 23 & 10 & 18 \\
& 0.60 & 61 & 77 & 4 & 2 \\
\hline \multirow{6}{*}{ PUC } & 0.54 & 757 & 2714 & 1169 & 288 \\
& 0.56 & 522 & 842 & 755 & 130 \\
& 0.58 & 160 & 251 & 576 & 438 \\
& 0.60 & 147 & 69 & 746 & 31 \\
\hline
\end{tabular}

